\title{
Singular Points and Projective Limits in Relativity
}

\author{
M. J. Slupinski ${ }^{1}$ and C. J. S. Clarke ${ }^{2}$ \\ Departments of Mathematics, ${ }^{1}$ University of Lancaster, Lancaster LA1 4YL, and ${ }^{2}$ University of York, \\ York YO1 5DD, UK
}

\begin{abstract}
We describe a modification of Schmidt's b-boundary for a spacetime, using a projective limit construction. The resulting boundary provides endpoints for all incomplete inextensible curves that are not totally or partially trapped, and every boundary point is an endpoint of such a curve. Boundary points are always Hausdorff separated from interior points, and the construction gives separate past and future singularities in the $k=+1, \Lambda=0$, Friedmann cosmology.
\end{abstract}

\section{Introduction}

The singularity theorems of Hawking and Penrose [1] have made it clear that general relativistic models of physically reasonable situations (e.g. of collapsing stars or the matter filled universe) are likely to contain timelike or null curves that cannot be continued to arbitrarily large or negative values of proper time (or affine parameter). The word "singularity" was used to denote this state of affairs, which corresponds physically to the strange idea of particles or photons suddenly ceasing to exist (or coming into being). One feels that there should be some "reason" for such a situation, and so, in view of the properties of known exact solutions, one is led to conjecture that in some sense the curvature of space-time becomes unbounded along such a curve, the unboundedness leading to a breakdown of general relativistic physics; or more generally it could be that the curvature becomes unbounded arbitrarily near the curve in some sense.

A way of mathematically formulating this idea is to define singular points, attached to the space-time of general relativity as a topological boundary, so that one can talk about the behaviour of physical quantities, such as curvature, in the limit as one approaches a singular point. Various definitions of such boundaries have been proposed, the most natural mathematically being the bundle boundary (b-boundary) of Schmidt [2]. The precise definition is given below, but roughly speaking, it attaches to a space-time $M$ a (topological) boundary $\partial_{b} M$ which provides an endpoint for each curve in $M$ that is incomplete in a generalised sense; 
and which is such that incomplete curves in $M$ that get "closer together" terminate at the same end point. The b-boundary has the following features:

(i) The definition involves only the connection on $M$, not the whole Lorentz metric and is applicable also to positive-definite Riemannian spaces in which case it reduces to the normal completion of such a space.

(ii) If $M$ is a space-time, each point of $M$ has a neighbourhood which contains no curves incomplete in the above generalised sense.

However it has the following drawbacks:

(a) In some space-times [3] some points of $\partial_{b} M$ are not Hausdorff separated from points of $M$ - their only neighbourhood in $M \cup \partial_{b} M$ being $\bar{M}$ (which makes it impossible to talk of approaching the "singular point");

(b) related to this is the fact [4] that in the $k=+1$ Friedmann cosmology, which has physically singular limits both in the past and in the future, the b-boundary gives just one singular point, representing both past and future singularities;

(c) not all the singular points are at the "edge" of space-time: there are some which can only be reached by trapped curves, which continually reenter a compact set.

A detailed survey of results on the b-boundary is given in [6].

In the present paper we give full details of a modification of the b-boundary briefly announced in [5] that preserves (i) and (ii) while avoiding (a), (b), and (c). The definition is given in Sect. 3 ; the basic properties are developed in Sect. 4, (a) and (b) being dealt with by Proposition 5, (c) by Proposition 6.

\section{Preliminary Definitions and Conventions}

By a space-time, we shall throughout mean a fourdimensional, connected, paracompact, Hausdorff, $C^{\infty}$ manifold equipped with a $C^{k}$ pseudo-Riemannian metric $(2 \leqq k \leqq \infty)$ of signature $(+,-,-,-)$. We shall think of $(M, g)$ as modelling our universe, so that $M$ should be non-compact and $(M, g)$ should be inextendible; but we shall not use these properties except insofar as the construction to be described becomes trivial when $M$ is compact. $L M$ will denote the bundle of pseudo-orthonormal frames over $M$; it carries a natural positive-definite metric inducing a topological metric $d$. The b-boundary closure $\bar{M}$ of $M$ [2] is constructed by forming the Cauchy completion $\overline{L M}^{\text {metric }}$ of $L M$ with respect to $d$, and then taking the quotient by a natural action of the Lorentz group, thus defining a projection $\pi: \overline{L M}^{\text {metric }} \rightarrow \bar{M}$. We set $\partial_{b} M=\bar{M} \backslash M$. A curve in $M$ is called b-inextendible if it is inextendible and its horizontal lifts in $L M$ have finite length in the Riemannian metric. Every such curve terminates at a point in $\partial_{b} M$. If $U \subseteq M$ is an open subspace of $M$ one can define a b-boundary closure of $U$, since $(U, g \mid U)$ is an (extendible) space-time, denoted by $\bar{U}$ distinguishing it from its topological closure with respect to $M$, denoted by $\bar{U}^{\text {top }}$.

\section{The Projective Limit Construction}

One can think of the b-boundary as formed by attaching an endpoint to every bextendible curve in $M$ and then identifying the endpoints of curves having lifts that 
approach each other arbitrarily closely. The unwanted points noted in Sect. 1 seem to arise from identifications between endpoints that one intuitively feels should be separated. Some of these identifications are no longer made if one deletes a compact subset from the interior of $M$. The idea of the projective limit construction is to delete successively larger compact sets and form a limit of the resulting boundaries. It can be split into several steps:

Let $(M, g)$ be a space time as above.

(A) Let

$$
\begin{aligned}
\mathscr{T} & =\{V: V \cong M, M \backslash V \text { compact }\} \\
& =\left\{V_{\alpha}: \alpha \in A\right\}
\end{aligned}
$$

for some indexing set $A$.

Now $M \in \mathscr{T}$, so let $M=V_{\omega}$.

Then (i) $A$ is partially ordered by

$\alpha \leqq \beta \Leftrightarrow V_{\alpha} \subseteq V_{\beta}$.

(ii) $\mathscr{T} \cup\{\emptyset\}$ is a topology on $M$.

If $\alpha \leqq \beta$ let $i_{\alpha \beta}: V_{\alpha} \rightarrow V_{\beta}$ be the inclusion map.

(B) (i) If $V_{\alpha} \in \mathscr{T}$ then $\left(V_{\alpha}, g \mid V_{\alpha}\right)$ is a (possibly disconnected) space-time. If $V_{\alpha}$ is connected then $V_{\alpha}$ is well-defined; and if not we define $\bar{V}_{\alpha}$ to be the disjoint union of the b-completions of it connected components. We note that if $\bar{V}_{\alpha}^{\text {top }}$ is the topological closure of $V_{\alpha}$ in $M$ then if $x \in \bar{V}_{\alpha}^{\text {top }} \backslash V_{\alpha}$, there is a point in $\bar{V}_{\alpha}$ corresponding to $x$ only if there is a rectifyable curve in $V_{\alpha}$ ending at $x$. If this is the case then there is certainly a b-finite curve in $V_{\alpha}$ ending at $x$ and so there is at least one point in $\bar{V}_{\alpha}$ corresponding to $x$ (see [1] p. 283). We shall denote the subset of $\bar{V}_{\alpha}$ consisting of such $x$ by $\overline{\bar{V}}_{\alpha}$.

(ii) If $\alpha \leqq \beta$ and $i_{\alpha \beta}: V_{\alpha} \rightarrow V_{\beta}$ is the inclusion, then we can extend it to a continuous map $\bar{i}_{\alpha \beta}: \bar{V}_{\alpha} \rightarrow \bar{V}_{\beta}^{\alpha}$ such that if $\alpha \leqq \beta \leqq \gamma$ then $\bar{i}_{\alpha \gamma}=\bar{i}_{\beta \gamma}{ }^{\circ} \bar{i}_{\alpha \beta}$.

This map is not necessarily injective.

(C) Regard $V_{\alpha}, \overline{\bar{V}}_{\alpha}$ as subsets of $\bar{V}_{\alpha}$ and regard $\bar{V}_{\alpha}$ as disjoint from $M$.

Let $i_{\alpha}: V_{\alpha} \rightarrow M$ include $V_{\alpha}$ in $M$. Define $M_{\alpha}$ to be the adjunction space of $M$ and $\bar{V}_{\alpha}$ via the map $i_{\alpha}$ as follows:

(i) Put a topology on $M \cup \bar{V}_{\alpha}$ by taking the disjoint union of the topologies on $M$ and $\bar{V}_{\alpha}$.

(ii) Define an equivalence relation on $M \cup \bar{V}_{\alpha}$ by:

$x \sim y \Leftrightarrow$ either $i_{\alpha} x=y$ or $i_{\alpha} y=x$.

Let $M_{\alpha}=M \cup \bar{V}_{\alpha} / \sim$, the factor space.

(iii) Topologise $M_{\alpha}$ by requiring that the topology on $M_{\alpha}$ is the finest for which the projection: $M \cup \bar{V}_{\alpha}^{\alpha} \rightarrow M_{\alpha}: x \rightarrow[x] \sim$ is continuous.

Then this space $M_{\alpha}$ consists of $M$ together with the endpoints of the b-inextendible curves of $V_{\alpha}$. b-inextendible curves not ending at points in the topological closure of $V_{\alpha}$ in $M$ have endpoints in $\bar{V}_{\alpha} \backslash V_{\alpha}$, regarded as a subset of $M_{\alpha}$ : b-extendible curves ending at points in $\bar{V}_{\alpha}$ have endpoints both in $\bar{V}_{\alpha} \backslash V_{\alpha}$ and in $M$, which are not Hausdorff separated (regarding everything as taking place in $M_{\alpha}$ ). 
From the construction one finds that:

(a) There is a map $\bar{r}_{\alpha}: \bar{V}_{\alpha} \rightarrow M_{\alpha}$ which is a homeomorphism onto its image.

(b) The is a map $m_{\alpha}: M \rightarrow M_{\alpha}$ which is homeomorphism onto its image.

(c) $\bar{r}_{\alpha} \bar{V}_{\alpha}, \bar{r}_{\alpha} V_{\alpha}, m_{\alpha} M$ are open in $M_{\alpha}$.

(D) If $\alpha \leqq \beta$, we have

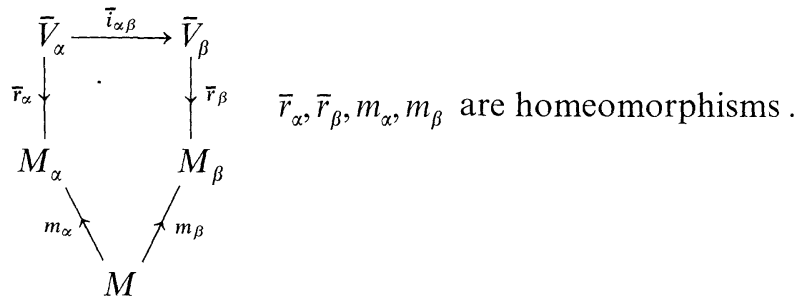

Define $\varrho_{\alpha \beta}: M_{\alpha} \rightarrow M_{\beta}$ by:

$$
\varrho_{\alpha \beta} x=\left\{\begin{array}{l}
\bar{r}_{\beta} \circ \bar{i}_{\alpha \beta} \circ \bar{r}_{\alpha}^{-1} x \text { if } x \in \bar{r}_{\alpha} \bar{V}_{\alpha} \\
m_{\beta} \circ m_{\alpha}^{-1} x \text { if } x \in m_{\alpha} M .
\end{array}\right.
$$

Then $\varrho_{\alpha \beta}$ is well-defined, continuous and if $\alpha \leqq \beta \leqq \gamma$ then

$\varrho_{\alpha \gamma}=\varrho_{\beta \gamma} \varrho_{\alpha \beta}$.

(E) Now we can take the projective limit of the system $\left\{M_{\alpha}, \varrho_{\alpha \beta}\right\}$ as follows:

$$
\begin{aligned}
M^{*} & =\lim \operatorname{proj}\left\{M_{\alpha}, \varrho_{\alpha \beta}\right\} \\
& \left.=\left\{\mathbf{x} \in \prod_{\alpha \in A} M_{\alpha}: \alpha \leqq \beta \Rightarrow \varrho_{\alpha \beta} x_{\alpha}=x_{\beta}\right\}\right\} .
\end{aligned}
$$

Let $\chi_{\alpha}: M^{*} \rightarrow M_{\alpha}: x \rightarrow x_{\alpha}$ be projection on the $\alpha^{\text {th }}$ coordinate. Topologise $M^{*}$ by requiring that it has the coarsest topology making each $\chi_{\alpha}$ continuous.

Finally we make the following definitions:

Definition 1. Let $M^{0}=\bigcap_{\alpha \in A} \overleftarrow{\chi}_{\alpha}\left(m_{\alpha} M\right)$

and

$$
\partial^{*} M=M^{*} \backslash M^{0}=\bigcup_{\alpha \in A}\left[M^{*} \backslash \overleftarrow{\chi}_{\alpha}\left(m_{v} M\right)\right] .
$$

$\partial^{*} M$ is the set whose elements we interpret as representing certain singularities in space-time.

\section{Basic Properties}

Proposition 1. (a) If $\mathbf{x} \in M^{*}$ and $x_{\omega}=m_{\omega} m$ for some $m \in M$, then $x_{\alpha}=m_{\alpha} m \forall_{\alpha} \in A$ and so $\mathbf{x} \in M^{0}$.

(b) If $\mathbf{x} \in \partial^{*} M$ then $x_{\alpha} \in \bar{r}_{\alpha} \partial_{b} V_{\alpha} \forall_{\alpha} \in A$ where $\partial_{b} V_{\alpha}=\bar{V}_{\alpha} \backslash \overline{\bar{V}}_{\alpha}$.

Proof. First we prove the following lemma:

Lemma. Suppose $V_{\alpha}, V_{\beta} \in \mathscr{T}$ with $\alpha \leqq \beta$ and that there is an $x \in V_{\beta}$ such that $x$ has an open neighbourhood $N$ with $N \cap i_{\alpha \beta} V_{\alpha}=\emptyset$. Then there is no $p \in \bar{V}_{\alpha}$ such that $\bar{i}_{\alpha \beta} p=x$. 
Proof. Suppose $\exists p \in \bar{V}_{\alpha}$ such that $\bar{i}_{\alpha \beta} p=x$.

Then $p \notin V_{\alpha}$.

There is a b-inextendible curve $\gamma:[0,1) \rightarrow \mathrm{V}_{\alpha}$ ending at $p$ whose image, $\bar{i}_{\alpha \beta}{ }^{\circ} \gamma$, defines a continuous $\bar{\gamma}:[0,1] \rightarrow V_{\beta}$ such that

(i) $\bar{\gamma}(1)=x$

(ii) $\bar{\gamma} \mid[0,1)=i_{\alpha \beta} \circ \gamma$.

Hence $\overleftarrow{\bar{\gamma}} N$ is open, contradicting $\overleftarrow{\bar{\gamma}} N=\{1\}$.

Proof. (cont.). (a) Assume $\exists \beta \in A$ such that $x_{\beta} \notin m_{\beta} M$. Choose $U$ open in $M$ such that $m \in U$ and $\bar{U}^{\text {top }}$ is compact.

Let

$$
V_{\eta}=\left(\left(M \backslash V_{\beta}\right) \cup \bar{U}^{\text {top }}\right) .
$$

Then

$$
\eta \leqq \beta \quad \text { so } \quad x_{\eta} \notin m_{\eta} M .
$$

Also

$$
\varrho_{\eta \omega} x_{\eta}=x_{\omega}=m_{\omega} m,
$$

so

$\bar{i}_{\eta \omega}\left(\bar{r}_{\eta} x_{\eta}\right)=m$ (definition of $\varrho$ maps).

But $U \cap V_{\eta}=\emptyset$ by construction and $m \in U$, so the lemma gives a contradiction. It follows easily that $x_{\beta}=m_{\beta} m \forall_{\beta} \in A$.

(b) $\mathbf{x} \in \partial^{*} M \Rightarrow x_{\alpha} \in \widetilde{V}_{\alpha} \backslash V \forall_{\alpha} \in A$ [by part (a)].

Suppose $\exists \beta \in A$ such that $x_{\beta} \in \bar{V}_{\beta} \cap\left(\bar{V}_{\beta} \backslash V_{\beta}\right)$; then $x_{\omega} \in m_{\omega} M$, and so $\mathbf{x} \in M^{0}$ by (a), a contradiction. Hence the result.

This proposition allows us to think of points in $M^{0}$ as sequences of constant projection and points in $\partial^{*} M$ as sequences of points in the "genuine" b-boundaries of the various $V_{\alpha}$.

Proposition 2. $\mathscr{B}=\left\{\chi_{\alpha} U: \alpha \in A, U \subseteq M_{\alpha}\right.$ is open $\}$ is a basis for the topology on $M^{*}$. Proof. First the following lemma:

Lemma. If $U=\bar{\chi}_{\beta} W$ where $\beta \in A$ and $W$ is open in $M_{\beta}$, then $\alpha \leqq \beta \Rightarrow \exists W^{\prime}$ open in $M_{\alpha}$ such that $U=\overleftarrow{\chi}_{\alpha} W^{\prime}$

Proof.

$$
\begin{aligned}
\alpha \leqq \beta & \Rightarrow \varrho_{\alpha \beta} \circ \chi_{\alpha}=\chi_{\beta} \\
& \Rightarrow \overleftarrow{\chi}_{\beta}=\overleftarrow{\chi}_{\alpha} \circ \overleftarrow{\varrho}_{\alpha \beta} .
\end{aligned}
$$

Put $W^{\prime}=\overleftarrow{\varrho}_{\alpha \beta} W \subseteq M_{\alpha}$ which is open by continuity.

Proof (cont.). Now it suffices to show that $\mathscr{B}$ has the finite intersection property Take $\overleftarrow{\chi}_{\alpha_{1}} U_{1}, \ldots, \overleftarrow{\chi}_{\alpha_{n}} U_{n}$ in $\mathscr{B}$. Let

$$
V_{\gamma}=V_{\alpha_{1}} \cap \ldots \cap V_{\alpha_{n}}\left(=M \backslash\left[V_{\alpha_{1}} \cup \ldots \cup V_{\alpha_{n}}\right] \in \mathscr{T}\right) \text {. }
$$

Then $\gamma \leqq \alpha_{i} i=1, \ldots, n$. 
Hence there is an open $U_{i}^{\prime} \subseteq M_{\gamma}$ for $i=1, \ldots$ such that $\overleftarrow{\chi}_{\gamma} U_{i}^{\prime}=\overleftarrow{\chi}_{\alpha_{i}} U_{i} i=1, \ldots$. Hence

$$
\bigcap_{i=1}^{i=n} \overleftarrow{\chi}_{\alpha_{i}} U_{i}=\bigcap_{i=1}^{i=n} \overleftarrow{\chi}_{\gamma} U_{i}^{\prime}=\overleftarrow{\chi}_{\gamma}\left(\bigcap_{i=1}^{i=n} U_{i}^{\prime}\right)
$$

But $\bigcap_{i=1}^{i=n} U_{i}^{\prime}$ is open in $M_{\gamma}$; whence the result.

Using these results and similar techniques one obtains (cf. [6] for details):

Proposition 3. $\forall \alpha, \chi_{\alpha} \mid M^{0}: M^{0} \rightarrow m_{\alpha} M$ is a homeomorphism with inverse $\overleftarrow{\chi}_{\alpha} \mid m_{\alpha} M$. Hence $M^{0}$ is homeomorphic to $M$.

Proposition 4. $M^{0}$ is dense in $M^{*}$.

These propositions mean that it is sensible to speak of $\partial^{*} M$ as a topological boundary to $M$.

Proposition 5. (a) If $\mathbf{x}, \mathbf{y} \in M^{*}$ and for some $\alpha \in A, x_{\alpha}, y_{\alpha}$ are Hausdorff separated, then $\mathbf{x}$ and $\mathbf{y}$ are Hausdorff separated.

(b) If $\mathbf{x} \in \partial^{*} M$ and $\mathbf{y} \in M^{0}$ then $\mathbf{x}$ and $\mathbf{y}$ are Hausdorff separated.

(a) Says, roughly, that if we can separate the endpoints of two curves in $M$ by the removal of a compact set, then in $M^{*}$ their endpoints, if they have endpoints, will be separated.

(b) Is a consequence of (a) and says that the boundary $\partial^{*} M$ is Hausdorff separated from the interior $M^{0}$. It follows that any continuous curve in $M^{0}$ which is totally trapped in a compact set cannot admit continuous extension to a point of $\partial^{*} M$.

The next proposition characterises the curves in $M^{0}(\approx M)$ which admit continuous extension to points in $\partial^{*} M$ and proves that all points in $\partial^{*} M$ are determined in this way.

Proposition 6. (i) If $\gamma$ is a b-finite inextendible curve in $M^{0}$ which is not totally or partially trapped in any compact subset of $M^{0}$, then $\gamma$ has an endpoint $\mathbf{x} \in \partial^{*} M$.

(ii) If $\mathbf{x} \in \partial^{*} M$ then $x$ is the endpoint of a b-finite inextendible curve in $M^{0}$ which is not totally or partially trapped in any compact subset of $M^{0}$.

Proof. First we note the following, the "universal" property of $M^{*}$.

If $X$ is any topological space and $\xi_{\alpha}: X \rightarrow M_{\alpha}$ are a system of continuous maps such that $\alpha \leqq \beta \Rightarrow \beta_{\beta}=\varrho_{\alpha \beta} \xi_{\alpha}$, then there is a unique continuous map $\xi: X \rightarrow M^{*}$ such that $\xi_{\alpha}=\chi_{\alpha} \circ \xi \forall_{\alpha} \in A$.

Proof of (i). Via the isomorphism $M^{0} \approx M, \gamma:[0,1) \rightarrow M^{0}$ defines $\gamma^{\prime}:[0,1) \rightarrow M$ such that, for all $\alpha \in A$, there is a $t_{\alpha} \in[0,1)$ such that $t>t_{\alpha} \Rightarrow \gamma^{\prime}(t) \in V_{\alpha}$ (regarded as a subset of $M$ ).

Therefore the curves $m_{\alpha} \circ \gamma^{\prime}:[0,1) \rightarrow M_{\alpha}$ admit continuous extension to $x_{\alpha}$ in $M_{\alpha} \backslash m_{\alpha} M$ with $\alpha \leqq \beta \Rightarrow Q_{\alpha \beta} x_{\alpha}=x_{\beta}$.

Let $\gamma_{\alpha}:[0,1] \rightarrow M_{\alpha}$ be these extensions. Then by the "universal" property above $\exists \Gamma:[0,1] \rightarrow M^{*}$ such that $\gamma_{\alpha}=\chi_{\alpha} \circ \Gamma$ and $\Gamma(1)$ is obviously an endpoint for $\gamma$ in $\partial^{*} M$ [in fact it is the sequence $\left(x_{\alpha}\right)_{\alpha \in A}$ ]. 
Proof. of (ii). Suppose $\mathbf{x} \in \partial^{*} M$. Let $x_{\omega}=p$.

Let $\left\{V_{n}\right\}_{n \in \mathbb{N}}$ be a countable collection of cocompact sets such that

(a) $V_{\alpha} \in T \Rightarrow \exists n \in \mathbb{N}$ such that $V_{\alpha} \supseteq V_{n}$,

(b) $m \geqq n \Rightarrow V_{m} \cong V_{n}$,

(c) $V_{0}=M$,

(d) $V_{n} \subseteq M \forall n \in \mathbb{N}$,

- such a collection exists since $M$ is paracompact. To avoid relabelling we assume that $\mathbb{N} \cong A$.

Then $x_{n}=\chi_{n} \mathbf{x} \in \partial_{b} V_{n}$ [by Proposition 1(b) and using the identification $\bar{r}_{n}^{-1}$ ]. Choose a b-inextendible (finite) $\gamma_{n}:[0,1) \rightarrow V_{n}$ ending at $x_{n}$. Lift $\gamma_{n}$ to $\bar{\gamma}_{n}$ in $L V_{n}$, ending at $\bar{x}_{n} \in \overline{L V}_{n}^{\text {metric }}$ in such a way that $m \geqq n \Rightarrow \bar{I}_{m n} \bar{x}_{m}=\bar{x}_{n}$ and let $\bar{x}_{0}=\bar{p}$, where $I_{m n}$ includes $L V_{m}$ in $L V_{n}$ and $\bar{I}_{m n}$ is its continuous extension.

Choose $X_{n}^{\prime}$ on $\bar{\gamma}_{n}$ such that $d_{n}\left(X_{n}^{\prime}, \bar{x}_{n}\right)<1 / 2^{n}$ where $d_{n}$ is the Schmidt metric on $L V_{n}$. Define $X_{n} \in L M=L V_{0}$ by $X_{n}=I_{n 0} X_{n}^{\prime}$. Then, we break down the rest of the argument in steps

(A) $d_{n}\left(X_{n}^{\prime}, I_{n+1, n} X_{n+1}^{\prime}\right) \leqq d_{n}\left(X_{n}^{\prime}, \bar{x}_{n}\right)+d_{n}\left(\bar{x}_{n}, I_{n+1, n} X_{n+1}^{\prime}\right.$,

$$
\begin{aligned}
& <1 / 2^{n}+d_{n+1}\left(\bar{x}_{n+1}, X_{n+1}^{\prime}\right) \\
& <1 / 2^{n-1} .
\end{aligned}
$$

So $\exists \Gamma_{n, n+1}^{\prime}:[1-1 /(\mathrm{n}-1), 1-1 / n] \rightarrow L V_{n}$ such that:

(i) $\Gamma_{n, n+1}^{\prime}(1-1 /(n-1))=X_{n}^{\prime}$ and $\Gamma_{n, n+1}^{\prime}(1-1 / n)=I_{n+1, n} X_{n+1}^{\prime}$,

(ii) Length $\Gamma_{n, n+1}^{\prime}<1 / 2^{n-1}$,

(iii) $y \in$ Image $\Gamma_{n, n+1}^{\prime} \Rightarrow$

$$
\begin{aligned}
d_{n}\left(y, \bar{x}_{n}\right) & \leqq d_{n}\left(y, X_{n}\right)+d_{n}\left(X_{n}, \bar{x}_{n}\right) \\
& <1 / 2^{n-1}+1 / 2^{n}<1 / 2^{n-2} .
\end{aligned}
$$

(B) Define $\Gamma_{k}:[1-1 /(k+1), 1) \rightarrow L V_{k}$ by $\Gamma_{k}(t)=I_{n k} \circ \Gamma_{n, n+1}^{\prime}(t)$ for

$$
t \in[1-1 /(n-1), 1-1 / n]
$$

as $n$ ranges over $k+2, k+3 \ldots$ and $k$ over $0,1,2,3, \ldots$.

Then:

$$
\text { Length } \begin{aligned}
\Gamma_{k} & \leqq \sum_{n \geqq k+2} \text { Length } \Gamma_{n, n+1} \\
& \leqq \sum_{n \geqq 2} \text { Length } \Gamma_{n, n+1} \\
& <\infty \text { by construction } .
\end{aligned}
$$

Also for $t \in[1-1 /(n-1), 1-1 / n]$ we have

$$
\begin{aligned}
d_{k}\left(\Gamma_{k}(t), \bar{x}_{k}\right) & <d_{n}\left(\Gamma_{n, n+1}(t), \bar{x}_{n}\right) \\
& <1 / 2^{n-2} \text { by (A) (iii). }
\end{aligned}
$$

So $\Gamma_{k}(t) \rightarrow \bar{x}_{k}$ as $t \rightarrow 1$.

Hence $\pi_{k} \circ \Gamma_{k}:[1-1 /(k+1), 1) \rightarrow V_{k}$ is a b-inextendible path ending at $\bar{\pi}_{k}\left(\bar{x}_{k}\right)$ $=x_{k} \in \bar{V}_{k}$ where $\bar{\pi}_{k}: \overline{L V}_{k}^{\text {metric }} \rightarrow \bar{V}_{k}$ is the extension of the bundle projection $\pi_{k}: L V_{k} \rightarrow V_{k}$. 
Let $\xi_{k}:[1-1 /(k+1), 1] \rightarrow \bar{V}_{k}$ be the continuous extension of $\pi_{k} \circ \Gamma_{k}$ such that $\xi_{k}(1)=x_{k}$ and define $\gamma_{k}:[0,1] \rightarrow M_{k}$ by:

$$
\gamma_{k}(t)=\left\{\begin{array}{lll}
m_{k} \circ \xi_{0} & \text { for } & t \in[0,1] \\
\bar{r}_{k} \circ \xi_{k} & \text { for } & t \in[1-1 /(k+1), 1] .
\end{array}\right.
$$

Then we have:

(i) $k \geqq j \Rightarrow \varrho_{k j} \gamma_{k}=\gamma_{j}$.

(ii) We can define $\gamma_{\alpha}:[0,1] \rightarrow M_{\alpha}$ for all $\alpha \in A$ (not just in $\mathbb{N} \subseteq A$ ) by picking $n \in \mathbb{N}$ such that $V_{n} \cong V_{\alpha}$ and defining $\gamma_{\alpha}=\varrho_{n \alpha} \gamma_{n}$. This is independent of the $n$ chosen by (i) immediately above.

The $\gamma_{\alpha}$ satisfy

(i) $\alpha \leqq \beta \Rightarrow \gamma_{\beta}=\varrho_{\alpha \beta} \gamma_{\alpha}$.

(ii) $\gamma_{\alpha}(1)=x_{\alpha}$.

Hence by the universality of $M^{*}$, there is a continuous map $\eta:[0,1] \rightarrow M^{*}$ such that $\forall \alpha \in A \quad \gamma_{\alpha}=\chi_{\alpha} \circ \eta$. Therefore $\eta \mid[0,1)$ has $\mathbf{x}$ as an endpoint and under the isomorphism $M^{0} \approx M$ is equal to $\xi_{0} \mid[0,1)$ which was b-finite and inextendible by construction.

(C) Now we need to show that $\eta \mid[0,1)$ is not totally or partially trapped in any compact subset of $M^{0}$. If it were then $\xi^{0} \mid[0,1)$ would be trapped in some compact subset $K$ of $M$.

Let

$$
V_{x}=M \backslash K \text {. }
$$

Choose $n \in \mathbb{N}$ such that $V_{\alpha} \supseteqq V_{n}$, then by construction $\xi^{0}(t) \in V_{n}$ for $1>t$ $\geqq 1-1 /(n-1)$, a contradiction to any kind of trappedness in $K$. [Here we regarded the $V_{\alpha}$ as subsets of $M$, unlike as in (A) and (B).]

\section{The Choice of a Boundary}

How adequately do the points of $\partial^{*} M$ represent singularities of space-time? In favour of $\partial^{*} M$ we can note that the arguments, that the b-boundary involves only the connection and does not introduce "internal" boundary points [(i) and (ii) of $\S 1]$, apply equally to $\partial^{*} M$; moreover $\partial^{*} M$ is free from the drawbacks we have noted for $\partial_{b} M[(\mathrm{a}),(\mathrm{b})$, and (c) of $\S 1]$. Also the two boundaries rank about equal in their ease of computation: $\partial^{*} M$ involves only local considerations (with respect to the topology $\mathscr{T} \cup\{\emptyset\}$ ), which is a simplification; but the topology of $\partial^{*} M$ is more involved. On the other hand, it could be argued against $\partial^{*} M$ that it is certainly outclassed by $\partial_{b} M$ in mathematical elegance.

We would hold that it is probably misguided to look for "the" perfect boundary for space-time. Instead, one should equip oneself with a range of possible boundaries so as to have tools available for various contingencies. A wide range of boundaries is provided by the following generalisation of the construction of Sect. 3. One starts with sets

$$
\{W \leqq M: W \text { open and } P(W)\}
$$


where $P$ is some property satisfying:

(a) For each $m \in M, \exists$ open $U \subseteq M$ and closed $V \subseteq M$ such that $m \in U \subseteq V$ and $P(M \backslash V)$.

(b) $P\left(W_{1}\right), \ldots, P\left(W_{n}\right) \Rightarrow P\left(W_{1} \cap \ldots \cap W_{n}\right)$.

(c) If we index the above family as $\left\{W_{\alpha}: \alpha \in A\right\}$ then there is a countable subset $\left\{Z_{i}: i \in \mathbb{N}\right\}$ with

(i) $Z_{0} \supseteqq Z_{1} \supseteqq Z_{2} \supseteqq \ldots$,

(ii) $P(X) \Rightarrow X \supseteqq Z_{n}$ for some $n \in \mathbb{N}$.

The boundary generated by applying the construction of Sect. 3 to such a collection of initial sets would then satisfy Propositions 1-6 appropriately modified if necessary. However, it might prove difficult to interpret such a boundary physically if $P$ is chosen so that $\{X: P(M \backslash X)\}$ do not correspond to a notion of "physically interor" sets.

Acknowledgements. M. J. Slupinski would like to thank Dr. C. T. J. Dodson and Dr. L. W. Flinn for many helpful discussions in the University of Lancaster, the Science Research Council for financial support, and the Université de Paris-Sud for hospitality 1978-79.

\section{References}

1. Hawking, S.W., Ellis, G.F.R.: The large scale structure of space-time. Cambridge: University Press 1974

2. Schmidt, B.G.: Gen. Rel. Grav. 1, 269-280 (1971)

3. Johnson, R.A.: J. Math. Phys. 18, 898-902 (1977)

4. Rosshard, B.: Commun. Math. Phys. 46, 263-268 (1976)

5. Clarke, C.J.S.: Gen. Rel. Grav. 10, 977-980 (1979)

6. Dodson, C.T.J.: Int. J. Theoret. Phys. 17, 389-504 (1978)

Communicated by R. Geroch

Received July 31, 1979 
